\title{
EGFR signaling in the HGG-02 glioblastoma cell line with an unusual loss of EGFR gene copy
}

\author{
JAN SKODA $^{1,2}$, JAKUB NERADIL ${ }^{1,3}$, KAREL ZITTERBART $^{2}$, JAROSLAV STERBA $^{2,3}$ and RENATA VESELSKA ${ }^{1,2}$ \\ ${ }^{1}$ Laboratory of Tumor Biology, Department of Experimental Biology, Faculty of Science, Masaryk University, Brno; \\ ${ }^{2}$ Department of Pediatric Oncology, University Hospital Brno and Faculty of Medicine, Masaryk University, Brno; \\ ${ }^{3}$ Masaryk Memorial Cancer Institute, Brno, Czech Republic
}

Received September 11, 2013; Accepted October 24, 2013

DOI: $10.3892 /$ or.2013.2864

\begin{abstract}
Epidermal growth factor receptor (EGFR) gene amplification and the overexpression of EGFR are described as common features of glioblastoma multiforme (GBM). Nevertheless, we previously reported the loss of EGFR gene copy in a GBM specimen from a patient with an unusually favorable course of the disease, and the HGG-02 cell line with this aberration was successfully derived from this tumor. Here, we present a detailed analysis of changes in gene expression and cell signaling in the HGG-02 cell line; the GM7 reference cell line with a standard EGFR gene copy number derived from a very aggressive GBM was used as a control. We confirmed the downregulation of EGFR expression and signaling in HGG-02 cells using different methods (RTK analysis, gene profiling and RT-PCR). Other changes that may have contributed to the non-aggressive phenotype of the primary tumor were identified, including the downregulated phosphorylation of the Axl and Trk receptors, as well as increased activity of JNK and p38 kinases. Notably, differences in PDGF signaling were detected in both of these cell lines; HGG-02 cells preferentially expressed and signaled through PDGFR $\alpha$, and PDGFR $\beta$ was strongly overexpressed and phosphorylated in the GM7 reference cell line. Using expression profiling of cancer-related genes, we revealed the specific profile of HGG-02 cells that included upregulated tumor-suppressors as well as downregulated genes associated with the extracellular matrix. This study represents the first comprehensive analysis of gene expression and cell signaling in glioblastoma cells with lower EGFR gene dosage. As indicated by our results, the TAM receptors, Trk receptors and PDGFRs need to be investigated further since their regulation appears to be important for glioblastoma biological features as well as the clinical course of the disease.
\end{abstract}

Correspondence to: Professor Renata Veselska, Laboratory of Tumor Biology, Department of Experimental Biology, Faculty of Science, Masaryk University, Kotlarska 2, 61137 Brno, Czech Republic E-mail: veselska@sci.muni.cz

Key words: glioblastoma multiforme, epidermal growth factor receptor signaling, PDGFR, TAM receptors, receptor tyrosine kinase, mitogen-activated protein kinase

\section{Introduction}

The epidermal growth factor receptor (EGFR) is a member of the EGFR receptor tyrosine kinase (RTK) family, together with ErB2/Neu/HER2, Erb3/HER3 and ErbB4/HER4 (1). Activating ligands of these receptors include epidermal growth factor (EGF), transforming growth factor $\alpha$ (TGF- $\alpha$ ), epiregulin, neuregulins and amphiregulin. Binding of the ligands to the extracellular domain of EGFR RTKs causes the formation of homodimers or heterodimers, cross-activation of tyrosine kinase domains and autophosphorylation. These events result in the stimulation of intracellular signaling cascades, including the phosphatidylinositol-3 kinase (PI3K)/Akt and mitogen-activated protein kinase (MAPK) cascades. Although EGFR signaling is essential for normal tissue development and homeostasis, the abnormal activity of EGFR RTK members plays a key role in tumor initiation and progression (2).

Glioblastoma multiforme (GBM) is the most frequent and malignant neoplasm of the human nervous system. Despite current therapies, the prognosis for patients with GBMs remains poor, with a median survival time that ranges from 8.8 months (patients $<50$ years of age) to 1.8 months (patients $>80$ years of age) $(3,4)$. EGFR gene amplification and EGFR overexpression are common features of GBM; however, they are rare in low-grade gliomas, which suggests a causal role of aberrant EGFR signaling in the pathogenesis of GBM (5). The amplification of EGFR is present in $35-70 \%$ of cases, typically in primary de novo GBM $(4,6,7)$.

In our previous study, we reported the unusual loss of $E G F R$ gene copy in GBM obtained from a patient with an unusually favorable course of the disease who experienced a survival of 5.5 years between diagnosis and death (8). Here, we present further detailed analysis of the HGG-02 cell line derived from this tumor when compared with the GM7 reference cell line that contains a standard EGFR gene copy number (9).

\section{Materials and methods}

Cell lines and cell culture. The HGG-02 (8) and GM7 (9) established glioblastoma cell lines were used in the present study. Cell cultures were maintained in DMEM supplemented with $20 \%$ fetal calf serum, $2 \mathrm{mM}$ glutamine and antibiotics (100 IU/ml of penicillin and $100 \mu \mathrm{g} / \mathrm{ml}$ of streptomycin) (PAA 
Laboratories, Linz, Austria) under standard conditions at $37^{\circ} \mathrm{C}$ in an atmosphere of $95 \%$ air and $5 \% \mathrm{CO}_{2}$. The cells were subcultivated once a week. Where indicated, the cells were starved in serum-free media.

$R T$-PCR. For RT-PCR, total RNA was extracted with the GenElute ${ }^{\mathrm{TM}}$ Mammalian Total RNA Miniprep kit (Sigma-Aldrich, St. Louis, MO, USA). For all samples, equal amounts of RNA ( $25 \mathrm{ng}$ of RNA/ $1 \mu \mathrm{l}$ of total reaction content) were reverse transcribed into cDNA using M-MLV (Top-Bio, Prague, Czech Republic) and oligo-dT (Qiagen Inc., Valencia, CA, USA) priming. Primers for EGFR, ErbB2, ErbB3, ErbB4, TGF- $\alpha$, EGF, epiregulin (EREG), neuroglycan C (NGC), NRG-1, NRG-2, PDGFR $\alpha$, PDGFR $\beta$, PDGFA, PDGFB, HSP90AB1 and GAPDH were used as described in Table I.

Phospho-RTK array analysis. The Human Phospho-RTK Array kit (R\&D Systems, Minneapolis, MN, USA) was used to determine the relative levels of tyrosine phosphorylation of 42 distinct RTKs according to the manufacturer's protocol. The arrays were incubated with $450 \mu \mathrm{g}$ of protein lysate. The levels of phosphorylation were quantified using ImageJ software and normalized to phosphotyrosine positive-control spots.

Phospho-MAPK array analysis. For the detection of the phosphorylation status of MAPKs and other serine/threonine kinases, the Human Phospho-MAPK Array kit (R\&D Systems) was used according to the manufacturer's protocol, and $250 \mu \mathrm{g}$ of protein lysate was used for each array. The arrays were analyzed using ImageJ software, and the levels of phosphorylation were normalized to positive control spots.

Expression profiling. Total RNA was extracted with the GenElute $^{\mathrm{TM}}$ Mammalian Total RNA Miniprep kit, and the samples were assessed spectrophotometrically. Purified biotin-labeled cRNA was generated using the Oligo GEArray ${ }^{\circledR}$ Reagent kit and was hybridized to the OHS-802 Oligo GEArray ${ }^{\circledR}$ Human Cancer Microarray, which profiles 440 genes related to cancer, in accordance with the manufacturer's instructions (SABiosciences, Frederick, MD, USA). The GEArray Expression Analysis Suite software (SABiosciences) was used for data analyses.

\section{Results}

EGFR and EGFRvIII mRNA status. As our previous findings suggested that the loss of the EGFR gene copy may have contributed to the unusually prolonged survival in a GBM patient (8), we continued a study of this case with a more detailed analysis of EGFR expression and dysregulation of cell signaling pathways in HGG-02 cells derived from the corresponding primary tumor tissue. Using RT-PCR, we assessed the EGFR status at the mRNA level in the HGG-02 and GM7 cell lines. HGG-02 cells showed significantly (2.24-fold) lower expression of EGFR when compared to the GM7 cells derived from a glioblastoma with a standard EGFR gene copy number. Relative expression levels of EGFR in HGG-02 and GM7 were 0.38 and 0.85 , respectively (Fig. 1). Furthermore, we examined the presence of the most common mutant form of EGFR, i.e., EGFRvIII. HGG-02 and GM7 cells did not express

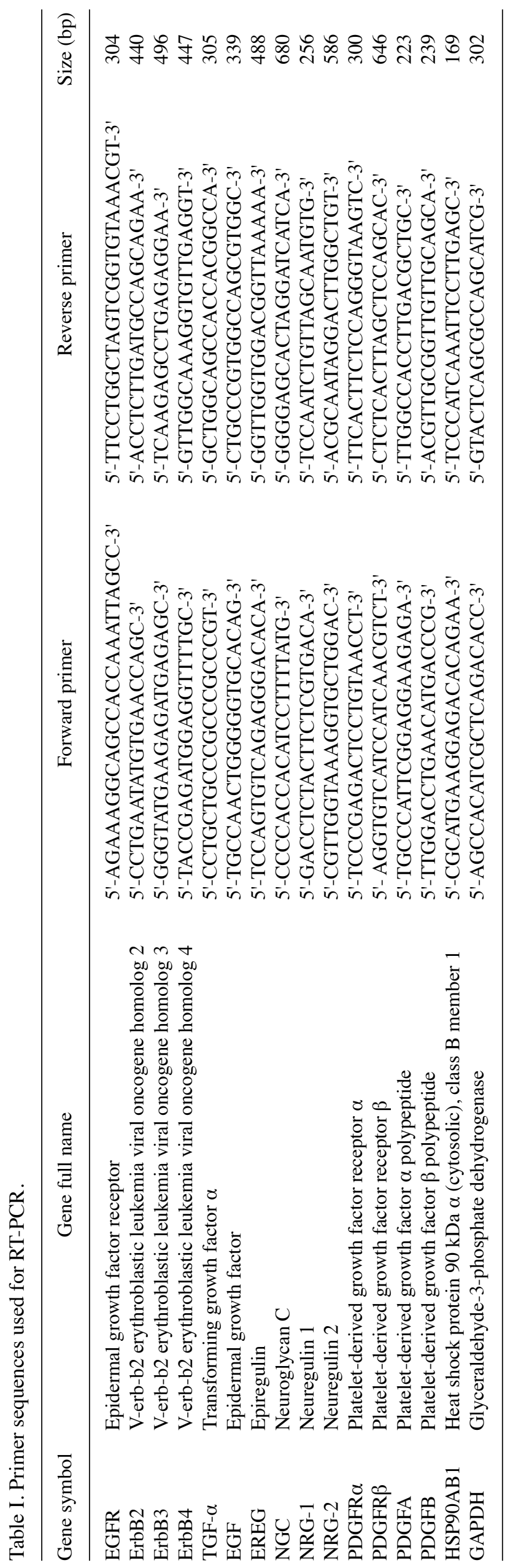




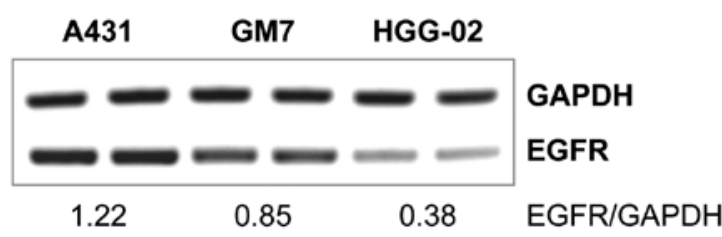

A

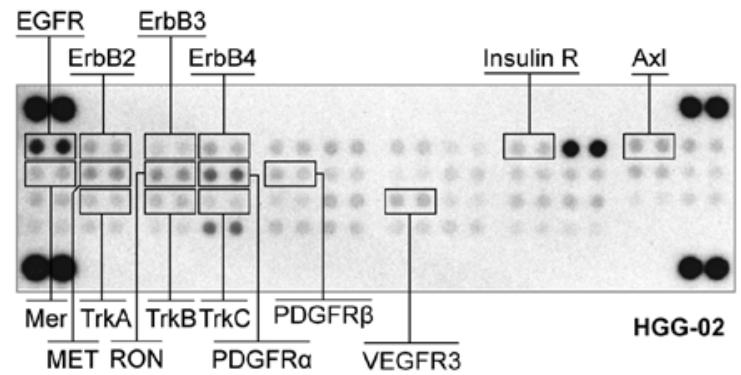

B

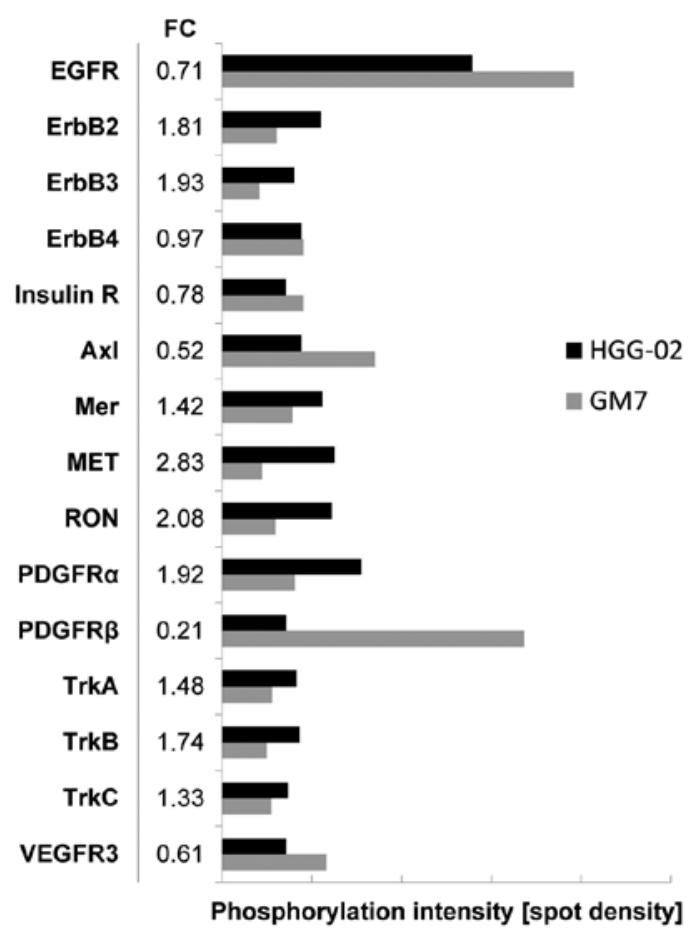

Figure 1. Expression status of EGFR in the HGG-02 and GM7 cell lines detected using semi-quantitative RT-PCR. Reduced expression of EGFR in HGG-02 cells was observed. The expression levels of EGFR were quantified with ImageJ software and normalized to the expression of the housekeeping gene, glyceraldehyde 3-phosphate dehydrogenase (GAPDH). Each sample was loaded in duplicate; EGFR/GAPDH ratios are noted. The A431 cell line served as the positive control; the GM7 cell line was derived from a glioblastoma with a standard EGFR gene copy number. EGFR, epidermal growth factor receptor.

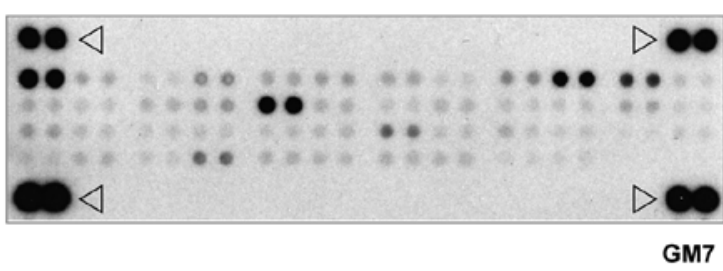

C



Figure 2. Phospho-RTK array in the HGG-02 cells demonstrates the downregulation of EGFR in comparison with the GM7 reference cell line. (A) Simultaneous detection of the phosphorylation status of 42 RTKs in the HGG-02 and GM7 cell lines using a human phospho-RTK array. Relevant receptors are noted. Phosphotyrosine-positive control spots are marked with an arrowhead. (B) In comparison with the GM7 cell line, HGG-02 cells exhibited downregulation of the EGFR, PDGFR $\beta$, Axl, VEGFR3 and insulin receptors. PDGFR $\alpha$, Mer, MET, RON, TrkA, TrkB and TrkC were upregulated. The level of phosphorylation was quantified using ImageJ software and normalized to phosphotyrosine-positive control spots. (C) The phosphorylation status after a 24-h serum-free cultivation. Note the overall higher phosphorylation levels and changes in ErbB2, ErbB3, Axl and Mer phosphorylation between the cell lines in comparison with the serum-containing conditions. FC, phosphorylation fold-change values of the HGG-02 cell line when compared to the GM7 reference cell line. RTK, receptor tyrosine kinase; EGFR, epidermal growth factor receptor.

the EGFRvIII variant (data not shown). These results support the hypothesis that the decreased EGFR mRNA expression in HGG-02 cells was caused by the lower EGFR gene dosage.

RTK signaling. As EGFR-downstream pathways may be activated and modulated by other RTKs aside from EGFR family receptors, we performed human phospho-RTK arrays to explore the phosphorylation status of 42 RTKs in the HGG-02 cells (Fig. 2A). Consistent with our previous data, we detected lower levels of phosphorylated EGFR in the HGG-02 cell line, which was in contrast with the GM7 cells (Fig. 2B). However, HGG-02 cells showed higher levels of ErbB2 and ErbB3 
A



B

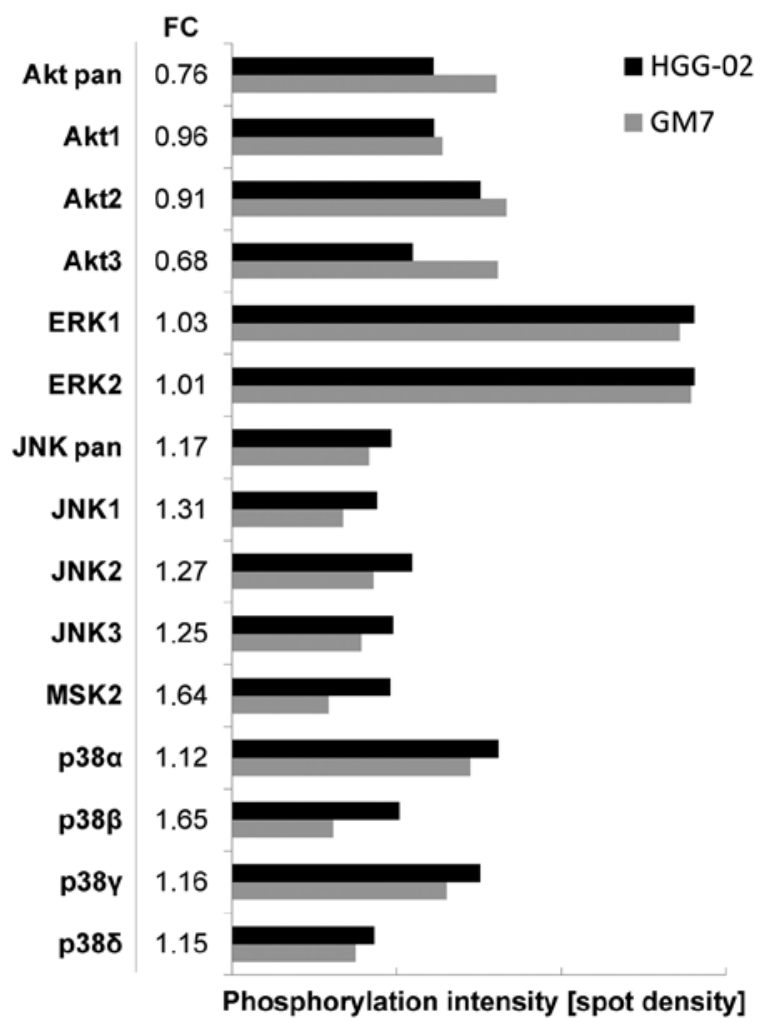

Figure 3. Phosphorylation status of MAPK in the HGG-02 and GM7 cell lines in (A) serum-containing and (B) serum-free conditions detected using phosphoMAPK arrays. The levels of phosphorylation were quantified using ImageJ software and were normalized to phosphotyrosine-positive control spots. FC, phosphorylation fold-change values of the HGG-02 cell line when compared to the GM7 reference cell line. MAPK, mitogen-activated protein kinase.

activation. ErbB4, another receptor of the EGFR family, did not show a significant difference in the phosphorylation state between these two cell lines.

In addition, we identified the downregulation of the phosphorylation of the PDGFR $\beta$, Axl, VEGFR3 and insulin receptors as well as the upregulation of the phosphorylation of PDGFR $\alpha$, MET, RON, Mer, TrkA, TrkB and TrkC in the HGG-02 cells (Fig. 2B).

To eliminate the influence of serum (which contains growth factors, hormones and other components) on the phosphorylation status of RTKs, we performed phospho-RTK arrays simultaneously using the cell lines after a 24-h serumfree cultivation. Notably, the serum-free conditions did not markedly change the phosphorylation status in the HGG-02 and GM7 cells; however, the overall level of phosphorylation was higher when compared to the serum-containing conditions (Fig. 2C). In the serum-free conditions, increased upregulation of Mer, ErbB2 and ErbB3 as well as downregulation of Axl were detected.

Signaling of MAPK and other serine/threonine kinases. To examine RTK downstream pathways activated in the HGG-02 cells, we employed phospho-MAPK arrays. We also performed the phospho-MAPK arrays on cells cultivated under serum-containing and serum-free conditions, as described above. An Akt pan-specific antibody revealed an overall diminished level of signaling through the Akt pathway in the HGG-02 cells when compared to the GM7 cells in the serum-containing and serum-free conditions (Fig. 3). However, differences in the phosphorylation levels of Akt1, Akt2 and Akt3 kinases were observed. Akt1 was more phosphorylated in the HGG-02 cells in the serum-containing but not in the serum-free conditions, whereas the levels of Akt2 and Akt3 phosphorylation were decreased regardless of the conditions. In addition, we detected an upregulation of signaling through the p38 pathway, JNK pathway and MSK2 in the HGG-02 cells. ERK1 and ERK2 signaling was also slightly elevated in the HGG-02 cells but only under the serum-containing conditions (Fig. 3A).

Expression of the EGFR and PDGFR families and their ligands. The results of the phospho-RTK arrays obtained under serum-free conditions indicated the presence of constitutively active receptor tyrosine-kinase pathways in the HGG-02 and GM7 cell lines. To investigate this possibility, we performed RT-PCR of the receptors in the EGFR and PDGFR families and their respective ligands after cultivation of the cells under serum-containing and serum-free conditions (Fig. 4).

As expected, expression of EGFR was decreased in the HGG-02 cells and did not significantly vary with time or under serum-free conditions. Although there was no difference in the expression of ErbB2, ErbB3 expression was higher under serum-free conditions and increased with time in both cell lines. ErbB4 expression was detected in the HGG-02 cells only and was stronger in the serum-free conditions. These patterns of expression were observable for its ligand 


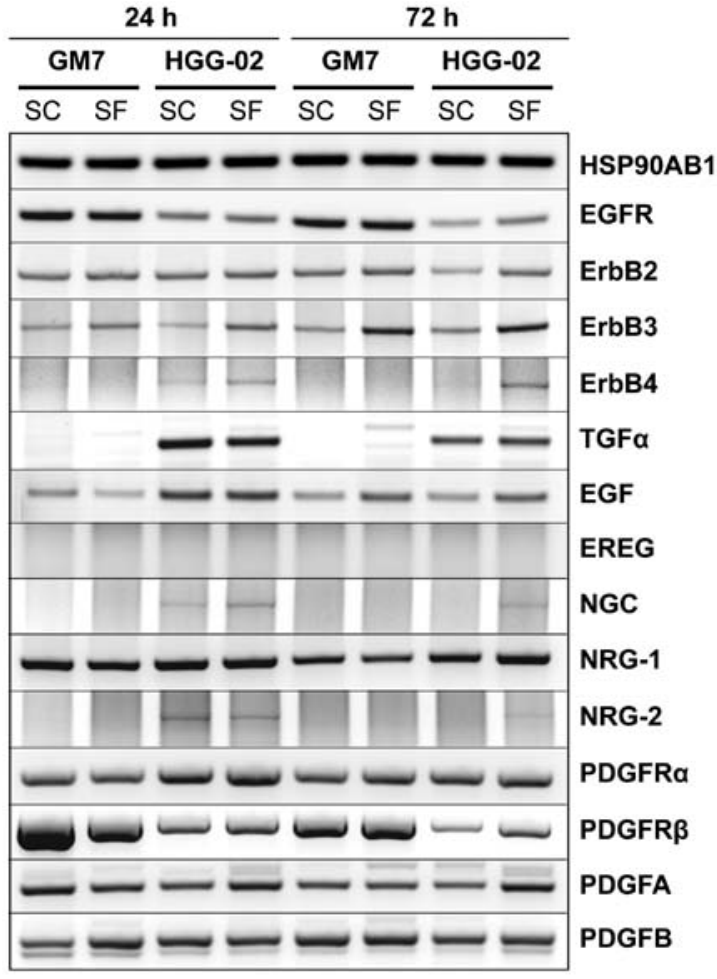

Figure 4. Expression of the receptors of the EGFR and the PDGFR families and their respective ligands using RT-PCR. The expression was analyzed after a 24- and 72-h culture in serum-containing and serum-free conditions. The housekeeping gene HSP90AB1 served as a control. SC, serum-containing conditions; SF, serum-free conditions.

NRG-2 and for the ErbB3-specific ligand NGC. In contrast to the low levels of EGFR mRNA, strong expression of the EGFR-specific ligand TGF- $\alpha$ was observed in the HGG-02 cells but not in the GM7 cells. EGF, the second EGFRspecific ligand, was also upregulated in the HGG-02 cells. Nevertheless, after $72 \mathrm{~h}$ of cultivation, the expression of EGF did not differ between cell lines and was higher in the serumfree conditions. Finally, the expression of NRG-1 increased in the HGG-02 cells after $72 \mathrm{~h}$ of cultivation, and EREG was not expressed in any of the samples.

We observed an apparent downregulation of PDGFR $\beta$ in the HGG-02 cells. In contrast, the expression of PDGFR $\alpha$ was slightly increased when compared with that in the GM7 cells. However, there were no obvious trends in the expression of the ligands for these receptors, including PDGFA and PDGFB.

Cancer-related gene expression. Gene expression profiling affirmed a decrease in EGFR expression at the mRNA level in the HGG-02 cells. Out of the 440 investigated genes, the HGG-02 expression profile revealed the upregulation of 108 genes (FC of 2-38.8) and downregulation of 10 genes (FC of 0.2-0.44) in comparison with the GM7 cells. Functional clustering showed that most of the upregulated genes are involved in the cell cycle, signaling pathways induced by growth factors, focal adhesions and apoptosis (Table II). The downregulated genes encode proteins responsible for organizing the extracellular matrix (ECM) or interacting with the ECM.

The 17 genes that were upregulated in the HGG-02 cells more than 5-fold were CDKN2A, TFAP2C, IGFBP3,
CDKN2B, FRZB, SRPX and Siva1. The downregulated genes included COL6A3, COL1A1, DCN, FN1, FBN1 and GDF15.

\section{Discussion}

Despite many advances in the treatment of GBM, the outcomes of patients with this tumor remain poor. Previously, we reported the case of a patient with GBM with a mild clinical course and a loss of EGFR gene copy (8). As both of these aspects are uncommon in GBMs, we performed a detailed analysis of the HGG-02 cell line that was derived from this patient's tumor to understand the cancer regulatory pathways that may have contributed to the favorable clinical course in this case. As control cells, the reference GM7 cell line that was derived from a highly aggressive tumor type and showed a standard $E G F R$ gene copy number (9) was used.

Using RT-PCR, a phospho-RTK array and expression profiling, we confirmed the downregulation of EGFR expression and signaling in HGG-02 cells. However, this downregulated signaling did not affect the downstream MAPKs, ERK1 or ERK2 and was possibly compensated for by the activation of the ErbB2 and ErbB3 receptors. These results are in agreement with another study that recently reported the compensatory activation of ErbB2 and ErbB3 receptors in GBM deprived of EGFR signaling, which suggests an intrinsic mechanism of GBM resistance to EGFR-targeted therapy (10). RT-PCR analysis revealed weak but detectable ErbB4 expression in the HGG-02 cells, while ErbB4 expression was not detected in the GM7 reference cell line. In contrast, the ErbB4 phosphorylation status was the same in these two cell lines, and we assume that this discrepancy may point to the previously questioned correlation of RNA and protein levels (11). However, the RT-PCR results of EGFR family-related ligands showed a strong elevation in TGF- $\alpha$, which was accompanied by an increased expression of EGF in the HGG-02 cells. Previous studies have suggested that EGFR and its ligands EGF and TGF- $\alpha$ play an important role in the autocrine/paracrine loop and support cell proliferation in human gliomas $(12,13)$. Nevertheless, considering that the HGG-02 cells acquired the loss of EGFR gene copy and the phospho-RTK array revealed a downregulation in EGFR signaling, we assume that the HGG-02 cells and the respective tumor do not represent EGFR-driven GBM.

Apart from the EGFR family, we identified changes in the phosphorylation of 11 other RTKs, including Axl. The phosphorylation of Axl was significantly reduced in comparison with the GM7 cells. This reduction was even more obvious under serum-free conditions, which suggests the constitutive activation of Axl in the GM7 reference cell line. Previous studies have found that Axl is constitutively phosphorylated in many glioma cell lines and that the downstream MAPK and PI3K pathways are activated (14). Moreover, Axl overexpression has been reported as a frequent event in human gliomas and is correlated with a poor prognosis in these patients (15). Therefore, the downregulation of Axl phosphorylation in HGG-02 cells may indicate a less aggressive phenotype of the respective tumor. Nevertheless, in the HGG-02 cells, we detected an upregulation of signaling through Mer, another member of the TAM family of RTKs (16). Recently, 2 independent studies demonstrated that Mer promotes invasion, 
Table II. Functional clustering of the molecular or biochemical pathways associated with genes that were differentially expressed in the HGG-02 cell line when compared to the GM7 cell line.

\begin{tabular}{lccc} 
Molecular or biochemical pathway & No. of deregulated genes & Percentage of deregulated genes & P-value \\
\hline Upregulated gene expression ( $>$ 2-fold change vs. GM7) & & & \\
Pathways in cancer & 20 & 19.05 & $<0.001$ \\
Cell cycle & 12 & 11.43 & $<0.001$ \\
MAPK signaling pathway & 12 & 11.43 & $<0.001$ \\
Glioma & 8 & 7.62 & $<0.001$ \\
Focal adhesions & 8 & 7.62 & 0.017 \\
Neurotrophin signaling pathway & 7 & 6.67 & 0.006 \\
p53 signaling pathway & 6 & 5.71 & 0.002 \\
Apoptosis & 6 & 5.71 & 0.006 \\
T cell receptor signaling pathway & 6 & 5.71 & 0.014 \\
B cell receptor signaling pathway & 5 & 4.76 & 0.017 \\
Toll-like receptor signaling pathway & 5 & 4.76 & 0.045 \\
Downregulated gene expression (<0.5-fold change vs. GM7) & & & \\
ECM-receptor interactions & 3 & 30.00 & 0.004 \\
Focal adhesions & 3 & 30.00 & 0.021 \\
\hline
\end{tabular}

MAPK, mitogen-activated protein kinase; ECM, extracellular matrix.

protects cells from apoptosis in GBM and has a similar function to Axl $(17,18)$. However, the reduction in Axl phosphorylation in the HGG-02 cells was 2 -fold greater than Mer upregulation. Overall, these results suggest that Axl and Mer play an important role in the aggressiveness of GBM and emphasize the need for further research of the TAM receptor tyrosine kinases in GBM.

Recently, it has been observed that MET, an HGF receptor, is activated during EGFR-targeted therapy resistance, and various studies have suggested that silencing EGFR is compensated by MET signaling in these cases (19). In accordance with these observations, we detected an upregulation of signaling through MET in the HGG-02 cells, which may partially compensate for the downregulation of EGFR signaling caused by the loss of EGFR gene copy. MET overexpression has been associated with a shorter survival time and poor response (20), and it has been suggested that MET activation is required for the acquisition of the glioblastoma cancer stem cell phenotype (21). Furthermore, upregulated phosphorylation of RON, the MSP receptor, may indicate another compensatory mechanism in HGG-02 cells. Eckerich et al reported that although RON signaling showed no mitogenic effect on GBM cells, it can induce glioma cell migration (22). However, these findings are in contrast with the unusually non-aggressive phenotype of the HGG-02 tumor, and we hypothesize that the activation of MET and RON signaling is not strong enough to overcome the loss of EGFR gene copy number in this case.

Nevertheless, HGG-02 cells exhibited increased phosphorylation of Trk receptors. It is well established that Trk receptors support the survival and differentiation of the nervous system; however, there is growing evidence that the Trk family can also induce or enhance cell death in certain tumor types, primarily in pediatric tumors of neural origin (23). It has been shown that expression of TrkA and TrkC correlates with a favorable outcome in neuroblastoma and medulloblastoma patients. In GBM, immunoreactivity for TrkA was shown to be inversely associated with the grade of malignancy (24). Moreover, TrkA overexpression was found to induce autophagic cell death that was mediated through the JNK pathway $(25,26)$. Therefore, we hypothesized that elevated signaling through Trk receptors and JNK MAPKs in HGG-02 cells contributed to the nonaggressive phenotype of the primary tumor.

Notably, we also detected an apparent difference in PDGF signaling in both of the cell lines studied. Whereas HGG-02 cells preferentially signaled through PDGFR $\alpha$, PDGFR $\beta$ was strongly phosphorylated in the GM7 reference cell line. Moreover, these results, which were obtained using the phospho-RTK array, corresponded with the results obtained by RT-PCR. Using RT-PCR, we detected PDGFR $\beta$ overexpression in the GM7 cells, whereas PDGFR $\alpha$ was overexpressed in the HGG-02 cells. Expression of PDGFs and PDGFRs has been observed even in low-grade gliomas, which suggests that this pathway possibly represents an early oncogenic event in gliomagenesis $(27,28)$. According to previous studies, PDGFR $\alpha$ is overexpressed in GBM cells, whereas PDGFR $\beta$ is detected in adjacent vascular cells (27). However, a recent study interrogated the proposed role of PDGFRs in intratumoral GBM heterogeneity (29). Kim et al found that expression of PDGFR $\beta$, but not PDGFR $\alpha$, was strongly associated with the glioblastoma stem cell (GSC) phenotype. Moreover, targeting PDGFR $\beta$ decreased GSC self-renewal, survival, tumor growth and invasion. Using in silico survival analysis, the authors noted that PDGFR $\beta$ indicated a poor prognosis, whereas PDGFR $\alpha$ was a positive prognostic marker. Together, these data support our observation in HGG-02 cells. Higher expression and signaling activity of PDGFR $\alpha$, instead of PDGFR $\beta$, may indicate the presence of a limited number of GSCs in the HGG-02 cell line when compared with the GM7 reference cell 
line derived from an aggressive tumor type. While our data stress the importance of PDGF signaling in GBM with respect to the distinct effects of PDGFR $\alpha$ and PDGFR $\beta$ in gliomagenesis, we strongly encourage further research of these RTKs in GBM biology.

We identified increased signaling through the p38 and JNK pathways in HGG-02 cells. It is known that p38 kinase activation is associated with anti-proliferative functions and plays a role in the induction of apoptosis (30). Moreover, recent studies have shown that $\mathrm{p} 38$ kinase activation is necessary for GBM cell death initiated by various anticancer agents (31-33). Furthermore, we detected increased signaling through the JNK kinases, which are also involved in apoptosis, and their activation is required for UV-induced apoptosis $(30,34)$. As previously noted, the JNK pathway can also mediate TrkA-induced autophagic cell death $(25,26)$. Overall, the increased activation of the p38 and JNK pathways, with regard to anti-proliferative and apoptotic functions, may represent the less aggressive characteristics of the HGG-02 cell line.

Although gene expression profiling detected changes in the expression of 118 genes, analysis of the most upregulated and downregulated genes in the HGG-02 cells revealed a specific expression profile of HGG-02 cells that may reflect the nonaggressive phenotype of this tumor. Seven of the 17 highly upregulated genes in the HGG-02 cells represent well accepted or potential tumor suppressors, including CDKN2A $(35,36)$, TFAP2C (37), IGFBP3 (38), CDKN2B (36), FRZB (39), SRPX (40) and Siva1 (41). However, the downregulated genes involved genes associated with the ECM, including COL6A3, COL1A1, DCN, FN1 and FBN1. These genes have been proposed to play an important role in controlling and facilitating the motility of glioma cells (42). For example, COL6A3 was found to correlate with glioma grade (43). Apart from the ECM-related genes, we detected significantly lower expression of GDF15 in the HGG-02 cells. Recent studies revealed that GDF15 contributes to the proliferation and immune escape of malignant gliomas $(44,45)$. This finding is in agreement with its low expression in the HGG-02 cell line that was derived from the unusually non-aggressive GBM.

In conclusion, the detailed analysis of the HGG-02 cell line confirmed the unusually reduced EGFR signaling and indicated several other targets that may contribute to the non-aggressive phenotype of the respective tumor. As suggested by our results, TAM receptors, Trk receptors and PDGFRs need to be further investigated since these proteins may play an important role in GBM biology, gliomagenesis and patient outcome.

\section{Acknowledgements}

The present study was supported by the European Regional Development Fund and the State Budget of the Czech Republic (RECAMO, CZ.1.05/2.1.00/03.0101) and OP VK CZ.1.07/2.3.00/20.0183.

\section{References}

1. Linggi B and Carpenter G: ErbB receptors: new insights on mechanisms and biology. Trends Cell Biol 16: 649-656, 2006.

2. Yarden Y and Sliwkowski MX: Untangling the ErbB signalling network. Nat Rev Mol Cell Biol 2: 127-137, 2001.
3. Ohgaki $\mathrm{H}$ and Kleihues P: Population-based studies on incidence, survival rates, and genetic alterations in astrocytic and oligodendroglial gliomas. J Neuropathol Exp Neurol 64: 479-489, 2005.

4. Ohgaki $\mathrm{H}$ and Kleihues P: Genetic pathways to primary and secondary glioblastoma. Am J Pathol 170: 1445-1453, 2007.

5. Hatanpaa KJ, Burma S, Zhao D and Habib AA: Epidermal growth factor receptor in glioma: signal transduction, neuropathology, imaging, and radioresistance. Neoplasia 12: 675-684, 2010.

6. Lopez-Gines C, Gil-Benso R, Ferrer-Luna R, et al: New pattern of EGFR amplification in glioblastoma and the relationship of gene copy number with gene expression profile. Mod Pathol 23: 856-865, 2010.

7. Bredel M, Scholtens DM, Yadav AK, et al: NFKBIA deletion in glioblastomas. N Engl J Med 364: 627-637, 2011.

8. Veselska R, Skoda J, Loja T, et al: An unusual loss of EGFR gene copy in glioblastoma multiforme in a child: a case report and analysis of a successfully derived HGG-02 cell line. Childs Nerv Syst 26: 841-846, 2010.

9. Loja T, Chlapek P, Kuglik P, Pesakova M, Oltova A, Cejpek P and Veselska R: Characterization of a GM7 glioblastoma cell line showing CD133 positivity and both cytoplasmic and nuclear localization of nestin. Oncol Rep 21: 119-127, 2009.

10. Clark PA, Iida M, Treisman DM, et al: Activation of multiple ERBB family receptors mediates glioblastoma cancer stem-like cell resistance to EGFR-targeted inhibition. Neoplasia 14: 420-428, 2012.

11. Gry M, Rimini R, Strömberg S, Asplund A, Pontén F, Uhlén M and Nilsson P: Correlations between RNA and protein expression profiles in 23 human cell lines. BMC Genomics 10: 365, 2009.

12. Tang P, Steck PA and Yung WK: The autocrine loop of TGF- $\alpha /$ EGFR and brain tumors. J Neurooncol 35: 303-314, 1997.

13. Ekstrand AJ, James CD, Cavenee WK, Seliger B, Pettersson RF and Collins VP: Genes for epidermal growth factor receptor, transforming growth factor $\alpha$, and epidermal growth factor and their expression in human gliomas in vivo. Cancer Res 51: 2164-2172, 1991

14. Stommel JM, Kimmelman AC, Ying H, et al: Coactivation of receptor tyrosine kinases affects the response of tumor cells to targeted therapies. Science 318: 287-290, 2007.

15. Hutterer M, Knyazev P, Abate A, et al: Axl and growth arrestspecific gene 6 are frequently overexpressed in human gliomas and predict poor prognosis in patients with glioblastoma multiforme. Clin Cancer Res 14: 130-138, 2008.

16. Linger RM, Keating AK, Earp HS and Graham DK: TAM receptor tyrosine kinases: biologic functions, signaling, and potential therapeutic targeting in human cancer. Adv Cancer Res 100: 35-83, 2008.

17. Rogers AE, Le JP, Sather S, Pernu BM, Graham DK, Pierce AM and Keating AK: Mer receptor tyrosine kinase inhibition impedes glioblastoma multiforme migration and alters cellular morphology. Oncogene 31: 4171-4181, 2012.

18. Wang Y, Moncayo G, Morin P Jr, et al: Mer receptor tyrosine kinase promotes invasion and survival in glioblastoma multiforme. Oncogene 32: 872-882, 2013.

19. Velpula KK, Dasari VR, Asuthkar S, Gorantla B and Tsung AJ: EGFR and c-Met cross talk in glioblastoma and its regulation by human cord blood stem cells. Transl Oncol 5: 379-392, 2012.

20. Kong DS, Song SY, Kim DH, et al: Prognostic significance of c-Met expression in glioblastomas. Cancer 115: 140-148, 2009.

21. Joo KM, Jin J, Kim E, et al: MET signaling regulates glioblastoma stem cells. Cancer Res 72: 3828-3838, 2012.

22. Eckerich C, Schulte A, Martens T, Zapf S, Westphal M and Lamszus K: RON receptor tyrosine kinase in human gliomas: expression, function, and identification of a novel soluble splice variant. J Neurochem 109: 969-980, 2009.

23. Harel L, Costa B and Fainzilber M: On the death Trk. Dev Neurobiol 70: 298-303, 2010.

24. Wadhwa S, Nag TC, Jindal A, Kushwaha R, Mahapatra AK and Sarkar C: Expression of the neurotrophin receptors Trk A and Trk B in adult human astrocytoma and glioblastoma. J Biosci 28: 181-188, 2003.

25. Hansen K, Wagner B, Hamel W, Schweizer M, Haag F, Westphal M and Lamszus K: Autophagic cell death induced by TrkA receptor activation in human glioblastoma cells. J Neurochem 103: 259-275, 2007.

26. Dadakhujaev S, Noh HS, Jung EJ, Hah YS, Kim CJ and Kim DR: The reduced catalase expression in TrkA-induced cells leads to autophagic cell death via ROS accumulation. Exp Cell Res 314: 3094-3106, 2008. 
27. Hermanson M, Funa K, Hartman M, Claesson-Welsh L, Heldin CH, Westermark B and Nistér M: Platelet-derived growth factor and its receptors in human glioma tissue: expression of messenger RNA and protein suggests the presence of autocrine and paracrine loops. Cancer Res 52: 3213-3219, 1992.

28. Maher EA, Furnari FB, Bachoo RM, Rowitch DH, Louis DN, Cavenee WK and DePinho RA: Malignant glioma: genetics and biology of a grave matter. Genes Dev 15: 1311-1333, 2001.

29. Kim Y, Kim E, Wu Q, et al: Platelet-derived growth factor receptors differentially inform intertumoral and intratumoral heterogeneity. Genes Dev 26: 1247-1262, 2012.

30. Wagner EF and Nebreda AR: Signal integration by JNK and p38 MAPK pathways in cancer development. Nat Rev Cancer 9 : 537-549, 2009.

31. Amantini C, Mosca M, Nabissi M, et al: Capsaicin-induced apoptosis of glioma cells is mediated by TRPV1 vanilloid receptor and requires p38 MAPK activation. J Neurochem 102: 977-990, 2007.

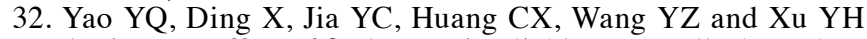
Anti-tumor effect of $\beta$-elemene in glioblastoma cells depends on p38 MAPK activation. Cancer Lett 264: 127-134, 2008.

33. Das A, Banik NL and Ray SK: Flavonoids activated caspases for apoptosis in human glioblastoma T98G and U87MG cells but not in human normal astrocytes. Cancer 116: 164-176, 2010.

34. Dhanasekaran DN and Reddy EP: JNK signaling in apoptosis. Oncogene 27: 6245-6251, 2008.

35. Liu W, Lv G, Li Y, Li L and Wang B: Downregulation of CDKN2A and suppression of cyclin D1 gene expressions in malignant gliomas. J Exp Clin Cancer Res 30: 76, 2011.

36. Feng J, Kim ST, Liu W, et al: An integrated analysis of germline and somatic, genetic and epigenetic alterations at 9p21.3 in glioblastoma. Cancer 118: 232-240, 2012.
37. Gabriely G, Yi M, Narayan RS, et al: Human glioma growth is controlled by microRNA-10b. Cancer Res 71: 3563-3572, 2011.

38. Wu C, Liu X, Wang Y, et al: Insulin-like factor binding protein-3 promotes the G1 cell cycle arrest in several cancer cell lines. Gene 512: 127-133, 2013.

39. Zi X, Guo Y, Simoneau AR, Hope C, Xie J, Holcombe RF and Hoang BH: Expression of Frzb/secreted Frizzled-related protein 3, a secreted Wnt antagonist, in human androgen-independent prostate cancer PC-3 cells suppresses tumor growth and cellular invasiveness. Cancer Res 65: 9762-9770, 2005.

40. Tambe Y, Isono T, Haraguchi S, Yoshioka-Yamashita A, Yutsudo M and Inoue H: A novel apoptotic pathway induced by the $d r s$ tumor suppressor gene. Oncogene 23: 2977-2987, 2004.

41. Li N, Jiang P, Du W, et al: Siva1 suppresses epithelial-mesenchymal transition and metastasis of tumor cells by inhibiting stathmin and stabilizing microtubules. Proc Natl Acad Sci USA 108: 12851-12856, 2011.

42. Mahesparan R, Tysnes BB, Read TA, Enger PO, Bjerkvig R and Lund-Johansen M: Extracellular matrix-induced cell migration from glioblastoma biopsy specimens in vitro. Acta Neuropathol 97: 231-239, 1999.

43. Fujita A, Sato JR, Festa F, et al: Identification of COL6A1 as a differentially expressed gene in human astrocytomas. Genet Mol Res 7: 371-378, 2008.

44. Wu A, Wei J, Kong LY, et al: Glioma cancer stem cells induce immunosuppressive macrophages/microglia. Neuro Oncol 12: 1113-1125, 2010.

45. Roth P, Junker M, Tritschler I, et al: GDF-15 contributes to proliferation and immune escape of malignant gliomas. Clin Cancer Res 16: 3851-3859, 2010. 